\title{
Estudio métrico del aporte de las universidades estatales costarricenses a los estudios de Género
}

José Pablo Meza Pérez

\begin{abstract}
RESUMEN
Los estudios métricos de información se han convertido en valiosos instrumentos para cuantificar y valorar el desarrollo de una disciplina o tema. Son de amplia difusión para el aseguramiento de la calidad e impacto en la investigación. En el campo de ciencias de información los estudios métricos han facilitado el desarrollo de nuevas disciplinas o objetos de investigación como la bibliotemetría, archivometría, cibermetría, informetría y muy recientemente la webmetría que es el estudio del comportamiento de los sitios web en la red de Internet.
\end{abstract}

Palabras claves: género, bibliometría, educación superior, Costa Rica.

\begin{abstract}
Metric Study of the Contribution of Costa Rican State Universities to Gender Studies. The metric studies of information have become valuable instruments to quantify and assess the development of a discipline or topic. They are widely spread out in order to assure the quality and impact on the research. In the field of information science the metric studies have facilitated the development of new disciplines or objects of research such as bibliometrics, archival science, cybermetrics, informetrics and most recently webometrics which studies the behavior of websites on the internet.
\end{abstract}

Key words: gender, bibliometrics, higher education, Costa Rica.

\section{Introducción}

La temática sobre género es un eje transversal en los procesos de enseñanza-aprendizaje en la Universidad Estatal a Distancia, posicionado en toda la actividad académica, de investigación y la extensión. Sus aristas para la investigación son tan abundantes que invitan a la reflexión a todas las disciplinas.

Las ciencias de la información son invitadas a realizar su aporte desde su objeto de estudio centrado en

Cátedra de Tecnologías de la Información. Escuela de Ciencias Sociales y Humanidades. Bachiller en bibliotecología y documentación de la Universidad Nacional. Licenciado en Administración Educativa de la Universidad Estatal a Distancia. Máster en Estudios de la Información del Sistema de Posgrado de la Universidad de Costa Rica. 
conocer el uso, consumo, producción y comunicación de la información e invitan a sus disciplinas como las computacionales, bibliotecologías, la documentación y la archivología. Ello les permite descubrir nuevas posibilidades de estudio, de investigación y conocimiento. Los aportes sobre los estudios de producción documental permiten la construcción de mapas del conocimiento que evidencian los recorridos y trazar nuevas rutas de desarrollo para nuevos temas o disciplinas.

La aplicación de las ciencias métricas al fenómeno de la producción bibliográfica ha permitido el seguimiento de nuevos campos de estudio como la bibliometría, la cibermetría, informetría, archivometría y muy recientemente la webmetría, esta como la disciplina que se encarga del estudio del comportamiento de los sitios web en la red de Internet. Estas disciplinas a la fecha cuentan con un buen número de indicadores cuantitativos, destacándose los aportes de sus propulsores y las leyes de Bradford, Lotka, Zip, Garfield y Price. Son utilizadas en la evaluación de la producción científica de gran vigencia para el aseguramiento de la calidad de la investigación. El Institute of Scientific Information, ISI de Filadelfia, fue pionero en los estudios de redes de citación y fuente de referencia para medir el impacto de la producción documental científica mundial.
Este aporte busca aplicar algunas de las leyes bibliométricas universalmente conocidas, y expresarlas en datos métricos, en torno al comportamiento cuantitativo y cualitativo de la producción bibliográfica en los estudios realizados por las universidades estatales sobre género desde su inicios a la fecha del segundo semestre del 2008.

\section{Materiales y métodos}

Este artículo sigue la estructura básica de los estudios métricos muy semejantes al esquema sugerido por las ciencias exactas y los estudios de enfoque positivista. Cuenta con una estructura conformada por: Introducción, descripción de los materiales, métodos utilizados, análisis de resultados, conclusiones y la bibliografía correspondiente.

Los datos para este estudio son parte de los resultados del Proyecto de Investigación del CIICLA, de la Universidad de Costa Rica, ejecutado por el autor de este artículo y divulgados a la comunidad universitaria como un aporte para potenciar los estudios de género como uno de los ejes transversales de la UNED, así como sugerir a los lectores nuevas líneas de investigación poco exploradas y evidenciar los puntos de mejora de la gestión de información en unidades especializadas para la investigación.

Se logró el acopio de toda la producción documental autóctona de 
10 unidades de información en el campo de la Ciencias Sociales y Letras de cuatro universidades estatales costarricenses; fue criterio condicional disponer de la información existente en los catálogos bibliográficos de las respectivas unidades de información, elaborando un listado de bibliografía tradicional conformado por: libros, informes de investigación, artículos de publicaciones periódicas, trabajos finales de graduación.

Para la construcción de los indicadores bibliométricos se utilizó la metodología de autoría y colaboración entre autores (Gorbea, 2005, 149 p.), cuantificando los siguientes datos para el análisis de resultados y conclusiones.

Se elaboraron siete indicadores bibliométricos: Índice de coautoría (IC). Tasa de documentos con coautoría (ITC). Índice de productividad de los autores (IP). Conteo de artículos (CA). Índices de categoría principal temática por artículo (ICPTA). Conteo geográfico de artículos (CGA) . Cantidad de artículos en publicaciones periódicas (CAPP).

Metodológicamente se encontró que no todas las unidades de información utilizan el mismo programa informático o los mismos términos de indización de la información y formato de descripción bibliográfica, lo que obligó a hacer procesos adicionales de importación de registros a una base de datos que compilara la totalidad de los registros; sin embargo, se comprobó que los datos existían pero se expresaban en formatos distintos. Para tales efectos se elaboran listados adicionales de autores y un vocabulario normalizado para el tratamiento temático.

Para efectos de presentación de resultados, serán sujeto de estudio cuatro unidades de análisis por registro bibliográfico (autoría, año de publicación, títulos de publicación periódica, categoría primaria temática).

Se elaboraron los siguientes reportes:

a. Reporte total de autores.

b. Reporte por año.

c. Reporte de títulos de publicaciones periódicas.

d. Reporte de categoría principal según siete áreas temáticas de especialidad del Programa de Géneros e Identidades del CIICLA.

\section{Análisis de resultados}

La Bibliografía sobre género de cuatro universidades estatales incluyó la revisión de los catálogos bibliográficos de las siguientes unidades de información.

\section{Universidad de Costa Rica:}

1. Sistema Información Bibliográfica Documentación e Información (SIBDI) agrupa la producción documental del Sistema de Estudios de Posgrado, Facultad de Derecho, Instituto 
de Investigaciones Psicológicas, y otras unidades académicas.

2. Biblioteca Eugenio Tortós, Biblioteca de la Facultad de Ciencias Sociales (BEFT).

3. Centro de Investigación en Identidad y Cultura Latinoamericanas (CIICLA).

4. Instituto de Investigaciones Sociales (IIS).

5. Centro de Investigaciones Históricas de América Central (CIHAC).

6. Centro de Investigación en Estudios de la Mujer (CIEM).

\section{Universidad Nacional:}

7. Sistema de Documentación de la Universidad Nacional (SIDUNA), que agrupa las referencias bibliográficas de las unidades, Instituto de Estudios Latinoamericanos (IDELA), Fondo
Bibliográfico del Instituto de Estudios de la Mujer, Posgrados de las facultades de Ciencias Sociales y de Letras.

8. Centro de Documentación de la Facultad de Ciencias Sociales.

\section{Universidad Nacional Estatal}

\section{a Distancia:}

9. Biblioteca Central, referencias de los programas de posgrado, Maestría en Violencia Social y Familiar.

\section{Instituto Tecnológico de}

\section{Costa Rica:}

\section{Biblioteca Central}

Se recuperó un gran número de registros bibliográficos sobre toda la producción autóctona en el campo de género en las cuatros universidades. Se identificaron en los catálogos

\section{Producción Documental por Universidad}

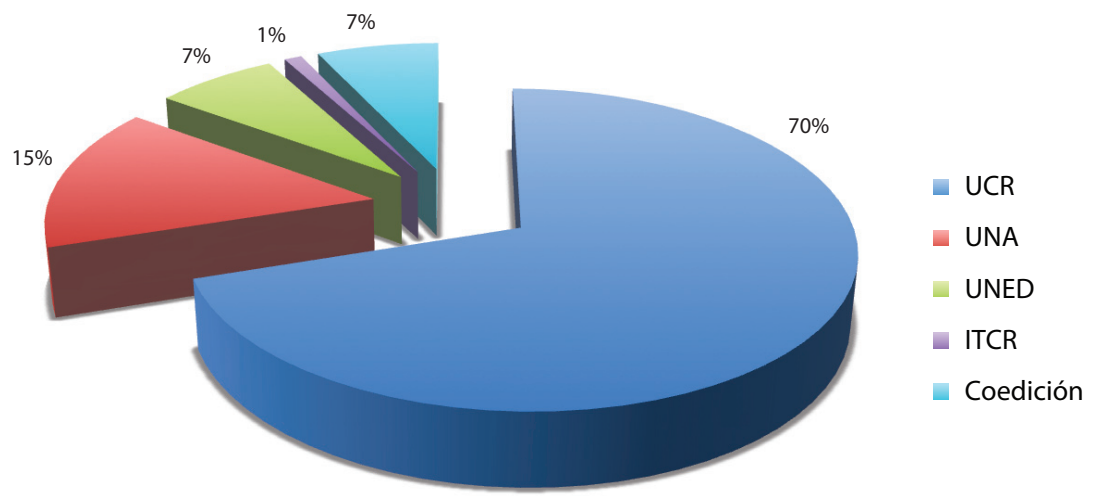

Fuente: Meza Pérez (2008). Informe de Investigación. 
bibliográficas 804 referencias bibliográficas de producción autóctona de las cuatros universidades, cronológicamente expresada en el rango de los años 1964 al 2008. Se debe aclarar que la investigación se realizó en el segundo semestre del 2008.

Todas las universidades han desarrollado estudios relacionados con el género. La Universidad de Costa Rica ha producido el $70 \%$ de la producción documental, seguida por la Universidad Nacional con un $15 \%$, la Universidad Estatal a Distancia el 7\% y el Instituto Tecnológico un 1\%.

Se encontró además un elemento que demuestra los esfuerzos de trabajo en conjunto en la figura de la publicación en coedición, "publicación entre dos o más entidades institucionales", con una participación del $7 \%$, esfuerzo realizado por la Universidad de Costa Rica y la Universidad Nacional.

La distribución de la población por tipo documental es la siguiente: $48,5 \%$ está conformado por trabajos finales de graduación, $26 \%$ por publicaciones monográficas e investigaciones y el $25,5 \%$ son artículos de publicación periódica.

\begin{tabular}{lc}
\hline Artículos de revistas & $25,5 \%$ \\
Trabajos Finales de graduación & $48,5 \%$ \\
Monografías e investigaciones & $26 \%$ \\
\hline
\end{tabular}

Fuente: Meza Pérez (2008). Informe de Investigación.
1. Índice de coautoría (IC). Es la totalidad de producción documental estudiada de 804 registros bibliográficos. El hallazgo más significativo encontrado fue identificar 1005 firmas: "Una firma es la representación de un autor". En términos generales existen 1005 autores que han conformado el universo del total de la producción documental autóctona de las cuatro universidades estatales costarricenses.

El índice de coautoría por autor es de 1,25 , estableciendo que mayoritariamente existe una tendencia a escribir individualmente; sin embargo, hay un aporte en coautoría que resulta significativo. Reflejando la posibilidad que el abordaje de la temática se realiza por grupos o equipos de trabajo.

\section{Tasa de documentos con} coautoría (TDC). La tasa de documentos con coautoría TDC o la proporción de documentos con autoría múltiple es 0,38 . El trabajo en colaboración con otros especialistas es importante para la productividad científica, como se muestra en el cuadro N. ${ }^{\circ} 1$.

El porcentaje de artículos con coautoría múltiple representa $38,04 \%$ del total de artículos. La forma o agrupación de mayor participación en coautoría múltiple es de dos autores con un $29,72 \%$, seguida por la 
TABLA 1

Total de firmas y representación porcentual

\begin{tabular}{ccc}
$\begin{array}{c}\text { Número de firmas } \\
\text { (autores firmantes del artículo) }\end{array}$ & Número de Firmas (autores) & Porcentaje \\
1 & & $61,94 \%$ \\
2 & 239 & $29,72 \%$ \\
3 & 48 & $5,97 \%$ \\
4 & 17 & $2,11 \%$ \\
5 & 2 & $0,24 \%$ \\
Total. & 804 & $99,98 \%$ \\
\hline
\end{tabular}

Fuente: Meza Pérez (2008). Informe de Investigación.

participación en coautoría múltiple con tres autores con 5,97\%. También la coautoría múltiple con cuatro autores es significativamente inferior pero su presencia es de $2,11 \%$.
3. Indice de productividad de los autores IP: calculado como el logaritmo en base 10 o ley de Lotka (Pulgarin, Parapeto y Cobos, 2004, Granda-. et al., 2006)

$\begin{array}{cccc}(\mathrm{n}) & \text { Númé } & \begin{array}{c}\% \\ \text { Índice de Productividad } \\ \text { de Lotka }(\lg 10 \mathrm{n})\end{array} \\ 1 & 890 & 88,55 \% & 0,0 \\ 2 & 86 & 8,61 \% & 0,2 \\ 3 & 15 & 1,49 \% & 0,3 \\ 4 & 4 & 0,39 \% & 0,4 \\ 5 & 5 & 0,49 \% & 0,5 \\ 6 & 3 & 0,29 \% & 0,6 \\ 8 & 1 & 0,09 \% & 0,8 \\ 13 & 1 & 0,09 \% & 1,3 \\ \end{array}$

Fuente: Meza Pérez (2008). Informe de Investigación.

Según el índice de productividad IP se puede observar en la tabla anterior que el $88,55 \%$ de los autores solamente han publicado un trabajo y son pequeños productores documentales. Se identificaron como medianos productores documentales todos aquellos autores cuya producción sea mayor a dos publicaciones y menor o igual a 9 publicación con un índice de Lotka expresado como $>0,2 \mathrm{y}=<0,9 \mathrm{y}$ conformado por el 
$11,31 \%$ del total de la población de autores de la población en estudio y solo se encontró una gran productora documental cuya producción es mayor a 10 publicaciones expresado en el índice de lotka como 1,3.

\section{Conteo anual de la produc-} ción documental: Este indicador representa el número de publicaciones producidas anualmente. Para el caso este estudio de la producción documental de cuatro universidades estatales costarricenses, disponible en 10 unidades de información, se ubica en el período cronológico de 1964 al 2008.

El promedio anual de publicaciones es de 18,2 documentos por año. Sin embargo, es notorio el

\section{Producción anual de documentos}

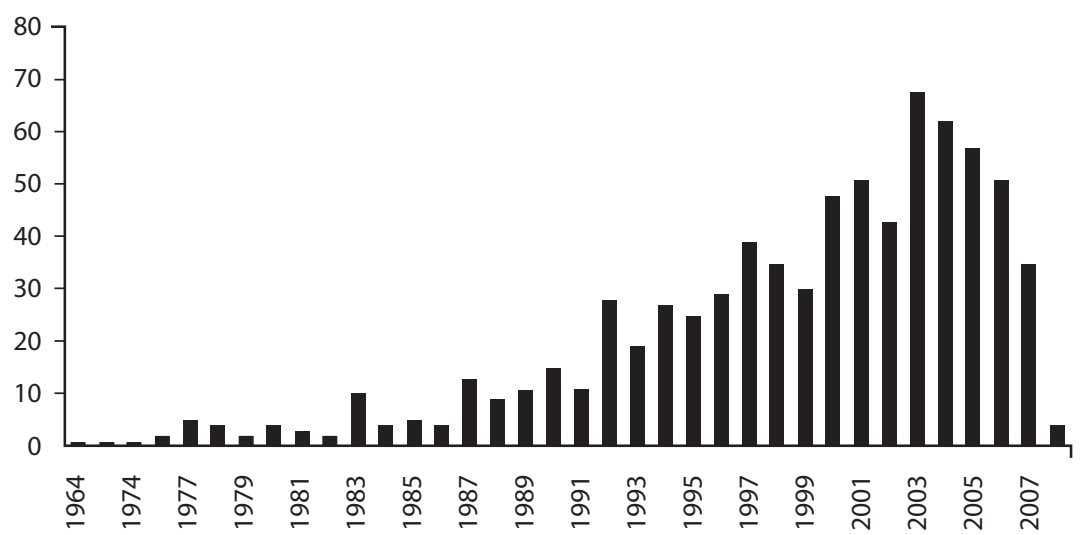

Fuente: Meza Pérez (2008). Informe de Investigación.

aumento de la producción documental en el período de 1977 al 2007 con ciclos de repunte de producción muy bien establecidos.

Se puede afirmar que existe un crecimiento sostenido de la producción documental en torno al objeto de estudio y en los 10 últimos años en el período de 1998 a 2007, produciendo el $64 \%$ del total de la producción universitaria estatal como se muestra en el siguiente gráfico, con un promedio anual de 48 nuevos documentos por año. 


\section{Producción Documental por Universidad}

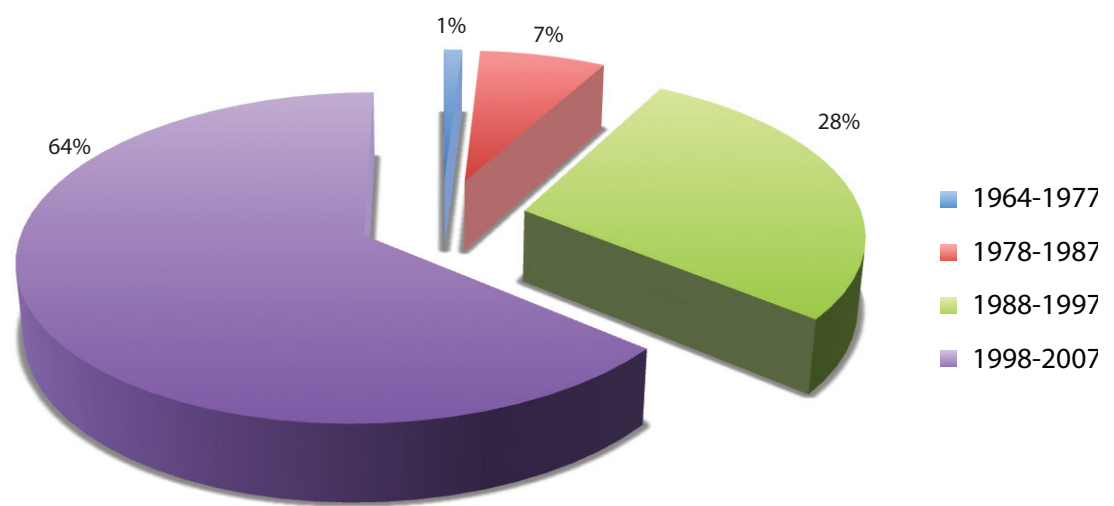

Fuente: Meza Pérez (2008). Informe de Investigación.

También se puede afirmar que es un área de estudio en expansión y en crecimiento con amplia recepción en la comunidad universitaria, que mejora la sensibilidad social de la población universitaria para la reflexión y estudio del tema. Contribuye para la sociedad costarricense con aporte y propuestas para mejorar el abordaje de la problemática.

Por estratos de población de interés en particular identidad femenina e identidad masculina presenta el siguiente comportamiento cronológico:

Para el estrato de identidad femenina su desarrollo cronológico inicia en el año 1977 y mantiene un promedio de 2,5 documentos anuales hasta el año 1994. Posterior a este período la producción documental aumenta a 10 documentos por año.

Para el estrato de población conformado por la categoría de identidad masculina la producción documental inicia en el año 1977, con un periodo significativo de discontinuidad en su producción anual hasta el año 1998. Para el año 2000 se puede cuantificar una producción documental más sostenida hasta la fecha. Sin embargo la producción no es superior a 3,75 artículos por año. 


\section{Identidad femenina producción documentación anual}

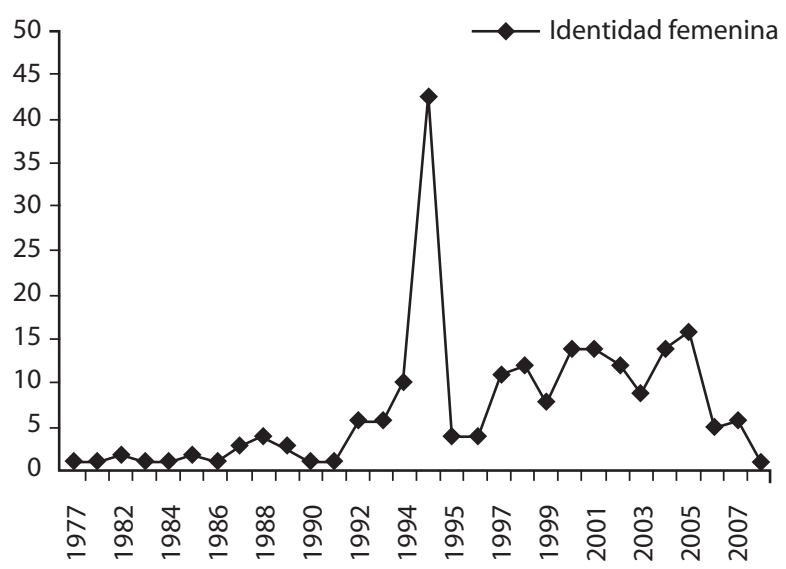

Fuente: Meza Pérez (2008). Informe de Investigación.

\section{Identidad masculina producción documentación anual}

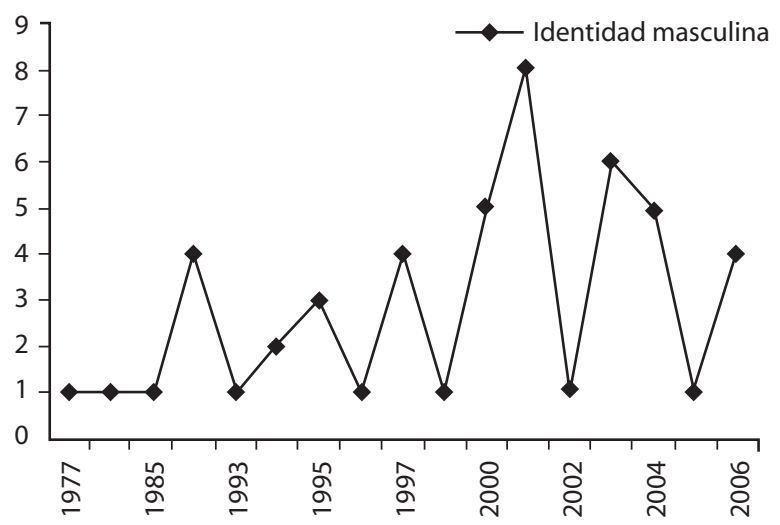

Fuente: Meza Pérez (2008). Informe de Investigación. 
5. Índice temático de los artículos. El contenido temático del total de la producción documental fue operacionalizado en un modelo basado en pocas variables que representan el contenido temático de la población para facilitar el proceso de estratificación y poder construir una inferencia que facilite la interpretación de la información.

Esta aproximación nos ha permitido construir seis categorías para evaluar el desarrollo temático de la población documental, reafirmando que en el período de 1964 al primer semestre del 2008 los estudios de género se han enfocado en el porcentaje que se muestra en el cuadro y gráfico seguidamente.

\begin{tabular}{lc} 
Categoría temática primaria & Porcentaje \\
\hline Género femenino & $30 \%$ \\
Identidad femenina & $23 \%$ \\
Identidad de géneros & $19 \%$ \\
Violencia contra el género & $18 \%$ \\
Identidad masculina & $7 \%$ \\
Sexualidad & $2 \%$ \\
Género masculino & $1 \%$ \\
Total & $100 \%$ \\
\hline
\end{tabular}

Fuente: Meza Pérez (2008). Informe de Investigación.

El período comprendido de 1998 al 2007 o producción documental de la última década estudiada, representa el $64 \%$ del total de población y el comportamiento mostrado es el siguiente.

Representación porcentual por categoría temática primaria: 1998-2007
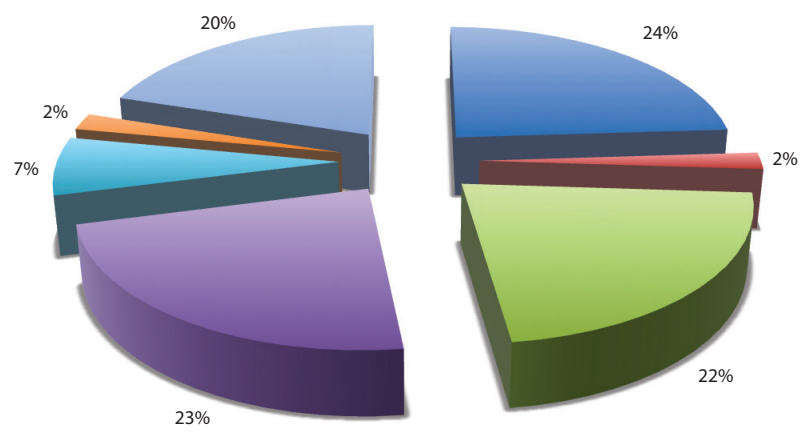

- Género femenino

- Género masculino

- Identidad de géneros

- Identidad femenina

- Identidad masculina

Sexualidad

Género masculino

Fuente: Meza Pérez (2008). Informe de Investigación. 


\begin{tabular}{lc} 
Categoría temática primaria & Porcentaje \\
\hline Género femenino & $24 \%$ \\
Identidad femenina & $23 \%$ \\
Identidad de géneros & $22 \%$ \\
Violencia contra el género & $20 \%$ \\
Identidad masculina & $7 \%$ \\
Sexualidad & $2 \%$ \\
Género masculino & $2 \%$ \\
Total & $100 \%$ \\
\hline
\end{tabular}

Fuente: Meza Pérez (2008). Informe de Investigación.

El segmento de población conformado por los estratos de identidad de géneros, identidad femenina y masculina representa el $48,13 \%$ de la población y su producción porcentual por tipo documental está conformada en un $49,3 \%$ por trabajos finales de graduación, $26,7 \%$ por monografías y $24 \%$ por artículos de publicaciones periódicas.

6. Conteo geográfico de artículos (CGA): La representación de la participación por área geográfica muestra la tendencia de que solo $11,19 \%$ de la producción documento se enfoca a estudiar un área geográfica en específico, y significativamente esos estudios se centraron en el gran área metropolitana y particularmente inferior la provincias costeras.

\section{Representación geográfica de la población}

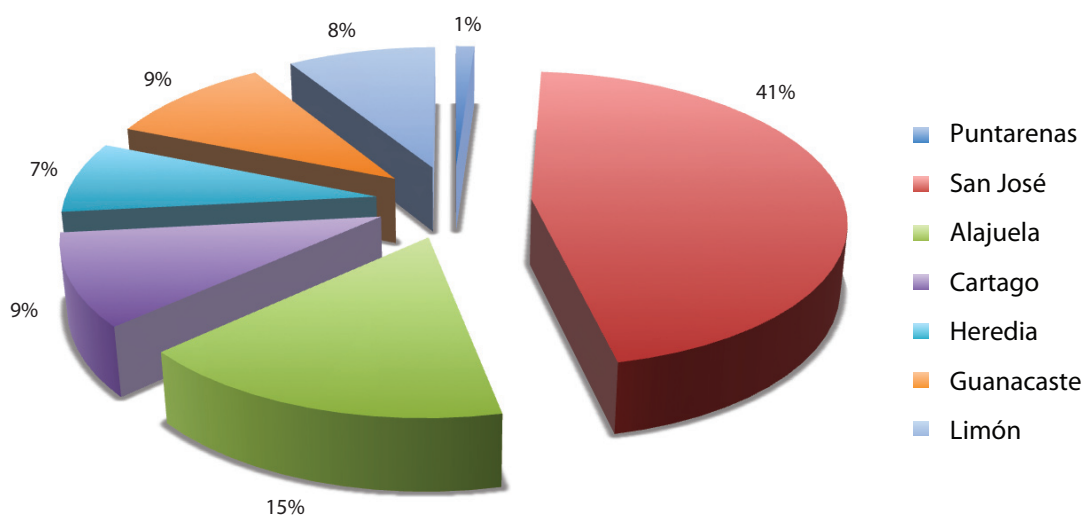

Fuente: Meza Pérez (2008). Informe de Investigación. 
7. Cantidad de artículos publicados en publicaciones periódicas. Las revistas, desde su aparición en el año de 1665 , son un recurso para la comunicación de la ciencia de gran versatilidad y de amplia difusión. Los trabajos bibliométricos de Bradford fueron pioneros en establecer la regularidades de especialización de las revistas. Sin embargo por el tamaño de la muestra estudiada para este caso no fue posible la aplicación de su ley para determinar el grupo de revista especializa- das de las universidades estatales en este campo. Pero sí fue posible establecer cuáles son las revistas universitarias que han publicado el mayor número de artículos en la especialidad de género, conformando un cuerpo editorial importante para lograr mayor visibilidad de la temática y de difusión.

El $25,37 \%$ de la población son artículos de publicaciones periódicas y destacándose 12 títulos de ellas que han realizado la mayor publicación de artículos en el campo.

$\begin{array}{lc}\text { Títulos de la publicaciones periódicas } & \text { Cant. Arts. } \\ \text { Revista de Ciencias Sociales } & 44 \\ \text { Diálogos Revista Electrónica de Historia } & 26 \\ \text { Anuario de Estudios Centroamericanos } & 18 \\ \text { Revista de filología y lingüística de la Universidad de Costa Rica } & 18 \\ \text { Revista de Historia } & 10 \\ \text { Revista Nacional de Cultura } & 9 \\ \text { Reflexiones } & 8 \\ \text { Repertorio Americano. Nueva Época } & 6 \\ \text { Contribuciones } & 5 \\ \text { Herencia } & 5 \\ \text { Serie Informes Finales de Investigación } & 5 \\ \text { Actualidades en Psicología } & 5 \\ \text { Fuente: Meza Pérez (2008). Informe de Investigación. } & \end{array}$

\section{Conclusiones y recomendaciones}

Los hallazgos en este proceso de investigación y análisis de resultados nos permiten llegar hacer las siguientes conclusiones y recomendaciones.
Gestión documental de las investigaciones en género

La producción documental sobre los estudios de género se encuentra dispersa en diferentes unidades de información, lo que requiere un 
esfuerzo adicional al investigador para realizar un recopilación exhaustiva de la información. La Bibliografía realizada en el proyecto de investigación Fondo de referencia sobre estudios de géneros desarrollado por el Programa Géneros e Identidades del CIICLA de la Universidad de Costa Rica es una herramienta importante para identificar los recursos y fuentes de información que sin duda agilizará significativamente esa tarea, y la misma se encuentra disponible en su Centro de Documentación.

Además de la evaluación hecha sobre población estudiada se encontró tres hallazgos adicionales de importancia para la gestión de la información.

No existe una normalización para el tratamiento temático de los estudios de género en las diferentes unidades de información.

Las unidades de información, los productores documentales e investigadores, deben realizar un esfuerzo por asegurar que su producción documental llegue a las unidades de información.

En particular se puede determinar que fue significativamente evidente los pocos hallazgos de dos tipologías documentales importantes para la investigación: informes de investigación y la llamada literatura gris. Se debe convertir en tarea prioritaria de los autores y dejar copia de sus trabajos en las bibliotecas, centros de documentación o sitios web que garanticen la custodia documental por un largo tiempo.

Para el caso de la UNED es evidente que se requiere completar la colección de la producción documental para visibilizar el aporte de la Universidad al tema.

El tratamiento de los recursos electrónicos o publicaciones electrónicas y recursos disponibles en Internet. Ninguna de las unidades de información procesa recursos en soporte electrónico. Solo se hace referencia si existe en versión impresa tradicionalmente en papel y se complementa con la dirección del recurso en el mejor de los casos. Se requiere ampliar la descripción, indización y recuperación de esta fuente de información que tiende a ser un recurso de información importante.

\section{Índice y tasa de documentos con coautoría}

El hecho que el 38,04\% se realice en forma conjunta, evidencia una tendencia a la formación de equipo de investigación y un tratamiento mayor al objeto de estudio. La formación de equipos de trabajo en la investigación facilita la especialización, busca un tratamiento más exhaustivo y especializado así como un escenario ideal para el trabajo en equipo, la confluencia de diferentes especialistas y un enfoque interdisciplinario. 
Índice de producción de autores

Los hallazgos realizados permiten identificar a un grupo de autores que han tenido mayor participación en la producción documental en las cuatro universidades estatales. También es significativo que exista un $11 \%$ de productores medianos de una población de 1005 autores, lo que demuestra un interés por darle un seguimiento al tema y presentar nuevos aportes al campo.

El hallazgo de encontrar un solo gran productor documental con 13 publicaciones por referencia con otros estudios métricos realizados por este autor. Los grandes productores documentales son muy escasos, y su presencia no es abundante en nuestro medio. La publicación científica no es una tarea fácil, requiere de un proceso de validación, evaluación por pares y de producción editorial. En otras ocasiones los grandes productores documentales se encuentran muy relacionados con las figuras de: editor, compilador o investigador destacado.

\section{Conteo anual de la producción documental}

El hallazgo más significativo en este apartado, es identificar a la última década como el período de mayor auge de la producción documental y la manifestación de períodos de repunte de la producción que supera al anterior, lo que refleja que el tema retoma vigencia con cierta periodicidad.

\section{Índice temático de los artículos}

Se puede afirmar que el comportamiento histórico de la temática de los estudios de géneros se mantiene. El $90 \%$ de producción documental se ubica en cuatro áreas principales de investigación: género femenino, identidad femenina, identidad de géneros y violencia contra el género. Las temáticas con menos desarrollo de producción documental son: identidad masculina, sexualidad y género masculino.

Es recomendable el realizar un seguimiento de los estudios de géneros en las universidades estatales para validar el comportamiento expresado en este estudio métrico y promover el desarrollo de las áreas de investigación identificadas con menor desarrollo y propiciar estudios en poblaciones específicas y en particular fuera de la gran área metropolitana como un mecanismo de inclusión social y validar la magnitud de la problemática.

Retomar los esfuerzos por parte de las unidades de información para reforzar los mecanismos de una efectiva gestión documental dentro de sus universidades para garantizar la custodia y resguardo de la producción documental autóctona. 


\section{Bibliografía}

Arencibia, J., Araújo Ruiz, J.A., Collymore Rodríguez, A., Leyva Rodríguez, Yadira. (2007) "Estudio Bibliométrico de la producción cientifica de la Revista CENIC Ciencias Químicas". 1996-2005. Revista CENIC Ciencias Químicas, Vol. 38, No. 1.

Díaz Mujica, D. (2007) “Análisis bibliométrico de la revista Archivos Latinoamericanos de Nutrición". An Venez Nutr, vol.20, no.1, p. 22-29.

Gorbea Portal, S. (2005). Modelo teórico para el estudio métrico de la información documental. Asturias, Trea.

Granda-Orive J. I. de, García-Río F., RoigVázquez F., Aleixandre-Benavent R., Valderrama-Zurían J. C., Martínez-Albiach J. M. et al. (2006). "Characterization of the leadership subject areas in the respiratory field in Spain". An. Med. Interna. Madrid. 23(11): 513-518.

ICAP. ( 2006). Estudio métrico y catálogo de la producción bibliográfica de Tesis de graduación del ICAP. San José, C.R. El Instituto.
ICAP. (2007). Red de gestión de información y conocimiento en administración pública. San José, C.R. El Instituto.

Meza Pérez, José Pablo. (2008) Informe final de investigación. "Fondo de referencia sobre estudios de géneros San Pedro, CR: $U C R$ ". Vicerrectoría de Investigación.

Pérez Andrés, C, Estrada Lorenz, J. M., Villar Álvarez, F., Rodríguez, J. R. (2002) Estudio bibliométrico de los artículos originales de la Revista Española de Salud Pública (1991-2000): Parte Primera indicadores generales. Rev. Esp. Salud Pública; 76: 659-672.

. (2003). Estudio bibliométrico de los artículos originales de la Revista Española de Salud Pública (1991-2000): Parte segunda. Rev. Esp. Salud Pública; 77: 333-346.

(2007). Estudio bibliométrico de los artículos originales de la Revista Española de Salud Pública (1991-2000): Parte tercera. Rev. Esp. Salud Pública, 81: 247-259. 
